\title{
Plasmablastic lymphoma phenotype is determined by genetic alterations in $M Y C$ and PRDM1
}

Santiago Montes-Moreno ${ }^{1,2}$, Nerea Martinez-Magunacelaya ${ }^{2}$, Tomás Zecchini-Barrese ${ }^{1}$, Sonia Gonzalez de Villambrosía ${ }^{3}$, Emma Linares ${ }^{1}$, Tamara Ranchal ${ }^{4}$, María Rodriguez-Pinilla ${ }^{4}$, Ana Batlle ${ }^{3}$, Laura Cereceda-Company ${ }^{2}$, Jose Bernardo Revert-Arce ${ }^{5}$, Carmen Almaraz ${ }^{2}$ and Miguel A Piris ${ }^{1,2}$

${ }^{1}$ Pathology Department, Servicio de Anatomía Patológica, Hospital Universitario Marqués de Valdecilla/ IDIVAL, Santander, Spain; ${ }^{2}$ Laboratorio de Genómica del Cáncer, IDIVAL, Santander, Spain; ${ }^{3}$ Hematology Department, Cytogenetics Unit, Hospital Universitario Marqués de Valdecilla/IDIVAL, Santander, Spain; ${ }^{4}$ Pathology Department, Fundación Jiménez Díaz, Madrid, Spain and ${ }^{5}$ Valdecilla Tumor Biobank Unit, HUMV/IDIVAL, Santander, Spain

Plasmablastic lymphoma is an uncommon aggressive non-Hodgkin B-cell lymphoma type defined as a highgrade large B-cell neoplasm with plasma cell phenotype. Genetic alterations in MYC have been found in a proportion $(\sim 60 \%)$ of plasmablastic lymphoma cases and lead to MYC-protein overexpression. Here, we performed a genetic and expression profile of $\mathbf{3 6}$ plasmablastic lymphoma cases and demonstrate that MYC overexpression is not restricted to MYC-translocated (46\%) or MYC-amplified cases (11\%). Furthermore, we demonstrate that recurrent somatic mutations in PRDM1 are found in $50 \%$ of plasmablastic lymphoma cases (8 of 16 cases evaluated). These mutations target critical functional domains (PR motif, proline rich domain, acidic region, and DNA-binding $\mathrm{Zn}$-finger domain) involved in the regulation of different targets such as MYC. Furthermore, these mutations are found frequently in association with $M Y C$ translocations (5 out of $9,56 \%$ of cases with MYC translocations were PRDM1-mutated), but not restricted to those cases, and lead to expression of an impaired PRDM1/Blimp1a protein. Our data suggest that PRDM1 mutations in plasmablastic lymphoma do not impair terminal B-cell differentiation, but contribute to the oncogenicity of $M Y C$, usually disregulated by $M Y C$ translocation or MYC amplification. In conclusion, aberrant coexpression of MYC and PRDM1/Blimp1a owing to genetic changes is responsible for the phenotype of plasmablastic lymphoma cases.

Modern Pathology (2017) 30, 85-94; doi:10.1038/modpathol.2016.162; published online 30 September 2016

Plasmablastic lymphoma is an uncommon aggressive non-Hodgkin B-cell lymphoma type defined as a high-grade large B-cell neoplasm with plasma cell phenotype (ie, loss of B-cell antigens with downregulation of CD20 and PAX5 expression, and overexpression of PRDM1/Blimp1 and $\mathrm{XBP}_{1} \mathrm{~s}^{1-4}$ ). Plasmablastic lymphoma was first described as an entity in 1997 and thought to be a human immunodeficiency virus-related lymphoma compromising the jaw and oral cavity. ${ }^{3}$ However, in the recent

Correspondence: Professor M-M Santiago, MD, PhD, Pathology Department, Servicio de Anatomía Patológica, Hospital Universitario Marqués de Valdecilla/IDIVAL, Cantabria, Avda de Valdecilla s/n, Santander 39010, España.

E-mail: smontes@humv.es

Received 4 June 2016; revised 1 August 2016; accepted 1 August 2016; published online 30 September 2016 years, a significant number of studies have demonstrated its association with other sources of immunodeficiency such as post-transplant setting, iatrogenic immunosuppression, as well as immunocompetent states in elderly patients. ${ }^{5-7}$ EBV infection, as demonstrated by in situ hybridization for EBV is found in the majority of the cases, but is not required for the development of a plasmablastic phenotype as clear-cut plasmablastic lymphoma can be found EBV-negative., ${ }^{3,4,8}$ In addition, recent evidence suggests that EBV or HIV status does not influence the gene expression profile patterns of plasmablastic lymphoma. ${ }^{9}$

Genetic alterations in $M Y C$ have been found in a proportion $(\sim 60 \%)$ of plasmablastic lymphoma cases, ${ }^{10,11}$ mainly consisting of translocations involving $M Y C$ and Ig. These translocations are not found in other lymphoma types with immunoblastic/ 
plasmablastic phenotype such as ALK+ large B-cell lymphoma, that may show another cytogenetic alterations involving $M Y C$ but lack $M Y C$ translocations. ${ }^{12}$ Gene expression analysis of plasmablastic lymphoma has found $M Y C$ overexpression at the mRNA level, and this overexpression is correlated with protein overexpression as detected by immunohistochemistry. ${ }^{9}$ Interestingly $M Y C$ overeexpression as detected by immunohistochemistry seems not to be restricted to those cases with MYC translocation by fluorescent in situ hybridization. ${ }^{12,13}$ All these data suggest that $M Y C$ overexpression has a central role in the pathogenesis of plasmablastic lymphoma. However, the mechanisms that lead to $M Y C$ overexpression in cases without $M Y C$ genetic translocations are poorly understood.

Blimp1 protein, encoded by PRDM1 gene is a tumor suppressor gene, ${ }^{14}$ and is considered the master regulator of plasma cell differentiation. ${ }^{15}$ Blimp1 protein is constitutively overexpressed by immunohistochemistry in the vast majority of plasmablastic lymphoma cases, frequently in concert with XBP1s, another gene involved in terminal B-cell differentiation. ${ }^{4,16}$ Recently, gene expression profiling data support the notion that the plasma cell differentiation program in plasmablastic lymphoma cases is due, as in normal plasma cells, to downregulation of BCR genes, and upregulation of both Blimp1 and XBP1s. ${ }^{9}$ Interestingly enough, in normal plasma cells, Blimp1, serves as a transcriptional repressor of $M Y C$ expression ${ }^{17}$ and is able to induce apoptosis by MYC downregulation in different lymphoma cell lines. ${ }^{17}$ Conversely, MYC protein is able to block terminal differentiation in several hematopoietic cell lines (cited in ref. 17). Thus, a regulatory loop involving MYC and Blimp1 is critical for the induction of a terminal differentiation program and growth arrest in normal B cells.

PRDM1 gene has been found mutated in $\sim 8 \%$ of overall diffuse large B-cell lymphoma. ${ }^{18,19}$ Furthermore, it has been found to be inactivated, either by mutation or genetic deletion of 6q21-q22.1 locus, in a significant fraction (23-24\%) of ABC-type DLBCL. ${ }^{14,20}$ Also transcriptional repression of PRDM1 by constitutively active translocated BCL6 (ref. 21) has been implicated in a significant fraction of DLBCL cases ( 21\%). Other studies have found genetic mutations of PRDM1 gene in 19\% (4 of 21) primary central nervous system lymphoma associated with abrogation of Blimp1-protein expression. ${ }^{22}$ Another study found loss of genetic material at $6 q 21$ in 10 out of $19(53 \%)$ cases of primary central nervous system lymphoma. However, sequencing of all exons of the PRDM1 gene failed to detect any mutations of the second allele in the tumors with heterozygous deletion of PRDM1 (ref. 23) in that series. Collectively, these studies show genetic inactivation of PRDM1 in a significant fraction of ABC-type DLBCL. These studies suggest that the genetic inactivation of PRDM1 may lead to a block in terminal B-cell differentiation with abrogation of Blimp1-protein expression, essential to the pathogenesis of ABC-type DLBCL. ${ }^{14,24}$ To date, however, the mutational genetic landscape of plasmablastic lymphoma is essentially unknown. ${ }^{25}$

Here, we demonstrate that PRDM1 genetic mutations (in particular, missense mutations) are highly prevalent and recurrent in plasmablastic lymphoma, and are associated with PRDM1/Blimp1 $\alpha$ expression, $M Y C$ translocation, $M Y C$ gain, and $M Y C$ overexpression at the mRNA and protein level. Interestingly enough, these PRDM1 mutations, unlike DLBCL NOS, are associated with a terminal B-cell differentiation profile, characteristic of plasmablastic lymphoma cases. Furthermore, PRDM1 mutations may be considered as a second-genetic hit in a significant proportion of plasmablastic lymphoma cases.

\section{Materials and methods}

\section{Case Selection}

Thirty-six cases diagnosed as plasmablastic lymphoma were retrieved from the files of the Pathology Department of the Hospital Universitario Marqués de Valdecilla. Formalin-fixed and paraffin-embedded tissue was available to construct a tissue microarray with 14 of these cases, following conventional protocols. ${ }^{26}$ Whole sections were used in the rest of the cases to perform the immunohistochemical, chromogenic in situ hybridization, and fluorescent in situ hybridization analysis. Clinical data were retrieved in all cases (see summary in Supplementary Table 1). The study and sample collection were approved by the local ethics committee (CEIC Cantabria) and complies with the Declaration of Helsinki.

\section{Immunohistochemistry and In situ Hybridization}

Immunohistochemical reactions were performed following conventional automated procedures. Primary antibodies against CD20 (DAKO, RTU), PAX5 (DAKO, RTU), CD138 (DAKO, RTU), CD38 (Leica, 1:200), IRF4/MUM1 (DAKO, RTU), Blimp1 (CNIO, 1:5), Kappa (DAKO, RTU), Lambda (DAKO, RTU), BCL6 (DAKO, RTU), BCL2 (DAKO, RTU), CD10 (DAKO, RTU), KI67 (DAKO, RTU), C-MYC (Abcam, 1:50), HHV-8 (Novus Biologicals, 1:10), EBV-LMP1 (DAKO, RTU), CD30 (DAKO, RTU), ALK (DAKO, RTU), p53 (DAKO, RTU) and p-STAT3 (Millipore $1: 100$ ) were used. A uniform threshold of $\geq 5 \%$ of the neoplastic population positive for the marker was considered to identify a positive case. Immunohistochemical stains were quantified and scored in 10\% increments. Chromogenic in situ hybridization for EBV-EBERs was carried out. EBV-EBER was considered positive when $\geq 10 \%$ of the large atypical cells were positive. Fluorescent in situ hybridization for the detection of $M Y C$ translocation was carried 
out using a Dual Color Break Apart Rearrangement probe involving the $M Y C$ gene region on chromosome 8q24 (Abbot Molecular). At least 10\% of the cells were required to be considered as positive for $M Y C$ break apart probes to identify a positive split signal. At least $15 \%$ of cells with extra copies of $M Y C$ gene were required to identify a case as positive for gains of $M Y C$ gene.

\section{Quantitative RT-PCR}

Primers for the quantification of MYC, PRDM1/ Blimp1 $\alpha$, and PRDM1/Blimp1 $\beta$ transcripts were designed, and RT-PCR was performed in 24 cases with available RNA from formalin-fixed paraffinembedded blocks. The sequence of the primers is as follows: MYC forward primer GAACCAGAGA AACCTAACAGTGC, reverse primer CGAAGCAGCT CTATTTCTGGA; PRDM1/Blimp1 $\alpha$ forward primer GATGGCGGTACTTCGGTTCA, reverse primer CCT CTTCACTGTTGGTGGCAT; PRDM1/Blimp1 $\beta$ forward primer AAGATCTATTCCAGAGGGGAGCT, reverse primer ACATAGCGCATCCAGTTGCT.

\section{Next-Generation Sequencing Using Amplicon-Based Library Generation of MYC-Promoter and PRDM1- Exonic Regions}

DNA was extracted from diagnostic formalin-fixed paraffin-embedded samples using conventional protocols. All samples subjected to DNA extraction were required to have $>50 \%$ of large atypical cells.

Primers for amplification and sequencing were designed using ExonPrimer (http://ihg.gsf.de/ihg/ ExonPrimer.html), checked with the USCS Genome Browser PCR in silico tool, and purchased from Sigma-Aldrich. A targeted amplification protocol was applied using primers against the regulatory regions in $M Y C$ gene by Blimp1 ( $M Y C$ promoter) and all seven exons of PRDM1 (details available in Supplementary Table 2). After PCR amplification using Pfu DNA polymerase (Promega Corporation) combined with the specific oligonucleotides, 16 cases had amplified products in all targeted regions of both genes and were available for library preparation.

Amplicons from $M Y C$ and PRDM1 genes were amplified and indexed as follows: all amplicons from the same patient were mixed in a tube, $500 \mathrm{ng}$ of each DNA sample was repaired using NEBNext: Ultra End Repair/dA Tailing Module kit (Biolabs) and linked to a pair of adapters, the $3^{\prime}$ and $5^{\prime}$ ends, respectively. A pair of indexing primers was then bound to the adapters to allow subsequent identification of each sample. DNA was purified with Agencourt AMPure XP beads (Beckman Coulter), and $4 \mathrm{ng}$ of each DNA was sequenced by nextgeneration sequencing on a MiSeq Personal platform (Illumina).

\section{Sequencing Data Interpretation and Reporting}

Variants were detected using the Somatic Variant Caller Algorithm from Illumina with default filtering settings. A summarized list of 63 missense variants in PRDM1 gene and 54 silent variants in $M Y C$ promoter region were subjected to individual review using IGV software (Integrative Genomics Viewer, Broad Institute). ${ }^{27}$ Adequate variant coverage (read depth >2500) and variant fraction $\geq 5 \%$ were considered restrictive criteria to make a call. A total of 20 gene variants in 8 cases were finally considered for PRDM1. Thus, 8 out of $16(50 \%)$ cases analyzed had at least 1 missense mutation in PRDM1-exonic regions (range 1-6 mutations). Three algorithms were used to predict the functional consequences of the variants found, including SIFT (http://sift.bii. a-star.edu.sg/), Polyphen-2 (http://genetics.bwh.har vard.edu/pph2/), and Condel (http://bg.upf.edu/ fannsdb/). Also dbSNP (http://www.ncbi.nlm.nih. gov/SNP/), COSMIC (http://cancer.sanger.ac.uk/cos mic), and existing literature regarding PRDM1 mutations in DLBCL were considered. ${ }^{14,18-22,28}$

Ten silent variants were considered in $M Y C$ promoter region. Of these, 3 were transitions $(G>A$ and $\mathrm{C}>\mathrm{T}$ in 2 cases) and were excluded. Thus, 7 silent variants were found in $M Y C$-promoter region in 5 cases out of 15 finally sequenced (33\%; Supplementary Figure 1).

\section{Results}

Plasmablastic Lymphoma is Found in Association with HIV, Post-Transplant States, Previous Lymphoma, and Advanced Age

We retrieved 26 males (70\%) and 10 females with plasmablastic lymphoma. Eleven cases (31\%) were known HIV-positive individuals and 16 cases were negative. Among HIV-negative patients, two of the cases had a prior history of solid organ/bone marrow transplantation and three patients had a prior history of hematolymphoid neoplasm (1 B lymphoblastic leukemia Phi+ treated with bone marrow transplantation, 1 follicular lymphoma, and 1 chronic lymphocytic leukemia). Patients with HIV infection were younger (median, 36 years old; range, 29-57 years old) than patients without any previous history of immunosuppression (related to transplantation or previous lymphoma) (median, 66 years old; range, $45-92$; $t$-test $P$-value for the difference $2.75 \times 10^{-6}$ ). The four patients with a previous history of immunosuppression/lymphoma had a median age of 56 years old (range 49-64).

The majority of the cases (25 out of $36,70 \%$ ) were diagnosed at extranodal locations, whereas 11 (30\%) were primarily nodal at diagnosis. Frequent extranodal locations included head and neck region (10 cases), bone and soft tissues (5 cases), and gastrointestinal tract (4 cases). For the cases with available clinical data, the majority were found in 
Table 1 Summary of phenotype and fluorescent in situ hybridization results

\begin{tabular}{|c|c|c|}
\hline Protein & $\%$ Cases positive ( $\mathrm{N}$ cases positive/total number evaluated) & Comments \\
\hline CD20 & $0 \%(0 / 36)^{*}$ & ${ }^{*} 5$ cases weakly positive ( $<5 \%$ of cells) \\
\hline PAX5 & $13 \%(4 / 31)^{*}$ & $* 5$ cases weakly positive $(<5 \%$ of cells) \\
\hline CD138 & $61 \%(22 / 36)$ & \\
\hline CD38 & $59 \%(13 / 21)$ & \\
\hline Blimp1 & $80 \%(28 / 35)$ & \\
\hline $\mathrm{k} / \lambda$ & $100 \%(20 / 20)^{*}$ & $16 \mathrm{~K}, 4 \lambda .{ }^{*} 7$ cases without detectable light-chain expression \\
\hline CD10 & $26 \%(8 / 30)$ & $\begin{array}{l}5 \text { out of } 6(83 \%) \text { cases with CD10 expression and FISH data } \\
\text { avaliable had MYC translocation by FISH }\end{array}$ \\
\hline BCL6 & $16 \%(4 / 25)$ & \\
\hline MUM1 & $96 \%(26 / 27)$ & \\
\hline C-MYC IHC & $100 \%(35 / 35)$ & $91 \% \geq 40 \%$ of cells positive \\
\hline C-MYC & $46 \%(12 / 26)^{*}$ & *Positive for translocation, three additional cases gains \\
\hline \multicolumn{3}{|r|}{ 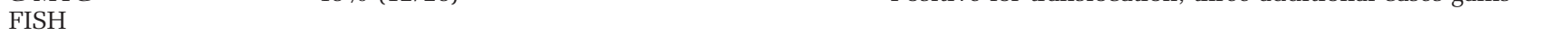 } \\
\hline BCL2 & $33 \%(7 / 21)$ & \\
\hline ALK & $0 \%(0 / 35)$ & \\
\hline HHV-8 & $0 \%(0 / 36)$ & \\
\hline EBV-EBER & $58 \%(21 / 36)$ & \\
\hline EBV-LMP1 & $6 \%(1 / 17)$ & \\
\hline CD30 & $29 \%(7 / 24)$ & \\
\hline STAT3-P & $82 \%(23 / 28)^{*}$ & ${ }^{*} 65 \%$ with $5-30 \%$ of cells positive \\
\hline ki67 & $91 \%(31 / 34)^{*}$ & ${ }^{*} \geq 70 \%$ of cells positive \\
\hline
\end{tabular}

advanced stage (III and IV, 15/20, 75\%) and high intermediate, high risk International Prognostic Index (11/18, 61\%; Supplementary Tables 1 and 3).

\section{Plasmablastic Lymphoma is Characterized by Large Blasts with a Phenotype of Plasma Cells, Very High Proliferative Activity, and Consistent Coexpression of MYC and Blimp1 proteins}

Results of the immunohistochemistry and fluorescent in situ hybridization results are shown in Table 1 and Figure 1. Consistent with the plasma cell differentiation profile, CD20 was completely negative in the vast majority of cases with only five cases with $<5 \%$ of cells with weak positivity. PAX5, another B-cell differentiation marker was negative in all but nine cases, five of which had $<5 \%$ of cells with weak positivity. Among plasma cell markers, MUM1 and Blimp1 showed higher sensitivity than CD138 and CD38 in the identification of terminal differentiation (96 and $80 \%$ of cases positive for MUM1 or Blimp1 compared with 61 and $59 \%$ for CD138 and CD38). EBV was found in $58 \%$ of the cases (21 out of 36) by EBV-EBER. EBER was positive in all 11 cases with HIV-positive status and 10 out of the rest of 25 cases $(40 \%)$. Of these 25 cases, 2 out of 4 patients with a prior history of $\mathrm{BM} /$ solid organ transplantation and/or lymphoma were EBVpositive. Thus, 8 patients without any known immunodeficiency status were EBV-positive (8 out of $21,38 \%$ ). Interestingly, 8 out of 30 cases studied showed expression of CD10. Light-chain restriction was identified in 20 cases by immunohistochemistry, with 7 cases showing absence of light-chain expression (20/27). Of note, HHV-8 was negative in all cases (0/36) and ALK was negative in all 35 cases tested. The case that was not tested was an 82-yearold male with lymphomatous pleural effusion, and only limited cytological material was available. Phosphorylated STAT3 protein was found overexpressed in $82 \%$ of the cases $(23 / 28)$. However, most of the cases had expression limited to $5-30 \%$ of the neoplastic population (65\% of the cases).

MYC protein was overexpressed in all cases tested, irrespective of translocation status (see later). The median expression in 35 cases tested was $80 \%$, with $91 \%$ of the cases with a percentage of positivity $\geq 40 \%$. Blimp1 was positive in $28 / 35$ cases $(80 \%)$. The median expression of Blimp1 was $100 \%$. Interestingly, MYC and Blimp1 were coexpressed in $80 \%$ of the cases. Ki67 was homogeneously high ( $\geq 70 \%$ in $91 \%$ of the cases), concordant with the MYC overexpression.

\section{Plasmablastic Lymphoma Carry Diverse Cytogenetic Abnormalities with Translocations, Gains, and Deletions of MYC Gene, but without Mutations in MYC-Promoter Region}

Fluorescent in situ hybridization analysis was available in 26 cases. Twelve of these cases (46\%) had MYC translocation. Three additional cases had MYC gains (11\%; Figure 1). One case had MYC deletion in one allele (case no. 23; Table 1 and Figure 2). EBV positivity was associated with $M Y C$ translocation and $M Y C$ gains $(57 \%$ of EBV-positive cases were $M Y C$-translocated/gained vs $20 \%$ of EBV-negative, $P<0.05)$. In relationship with CD10 expression, 5 out of 6 cases (83\%) with CD10 expression and fluorescent in situ hybridization data available had MYC translocation. These results are in line with previously published data showing an enrichment of CD10-positive and MYC-translocated cases in the immunoblastic variant of DLBCL. ${ }^{29}$

After targeted amplicon-based deep sequencing of $M Y C$-promoter region (the genomic region where 
a

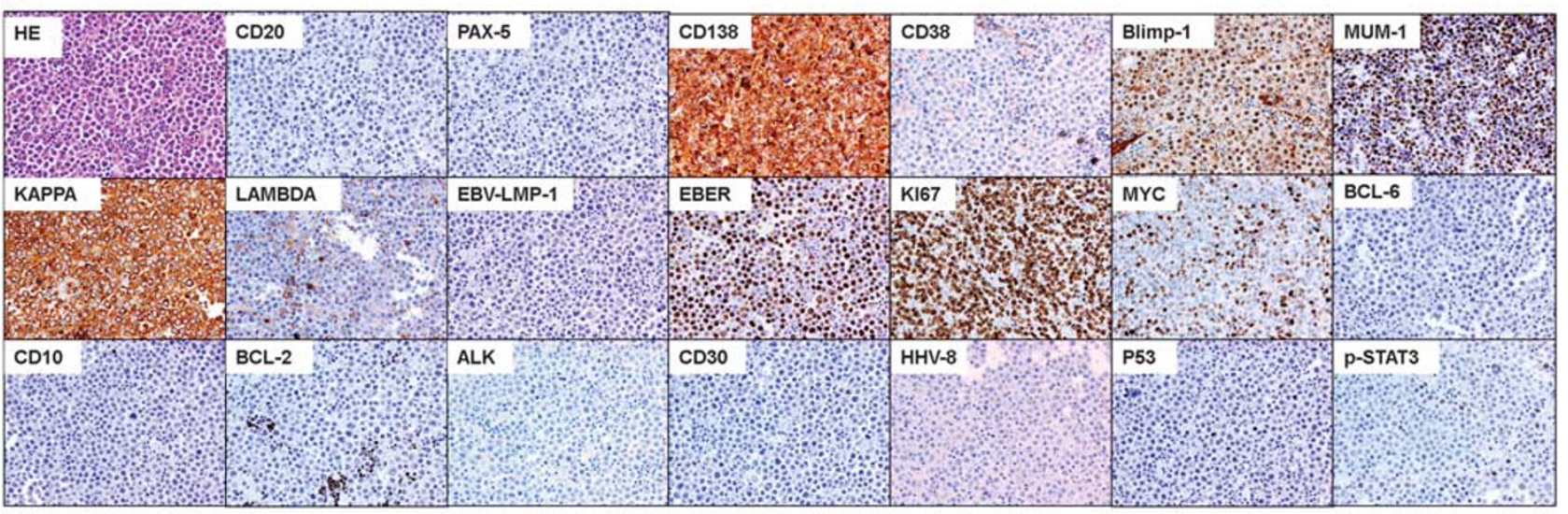

b

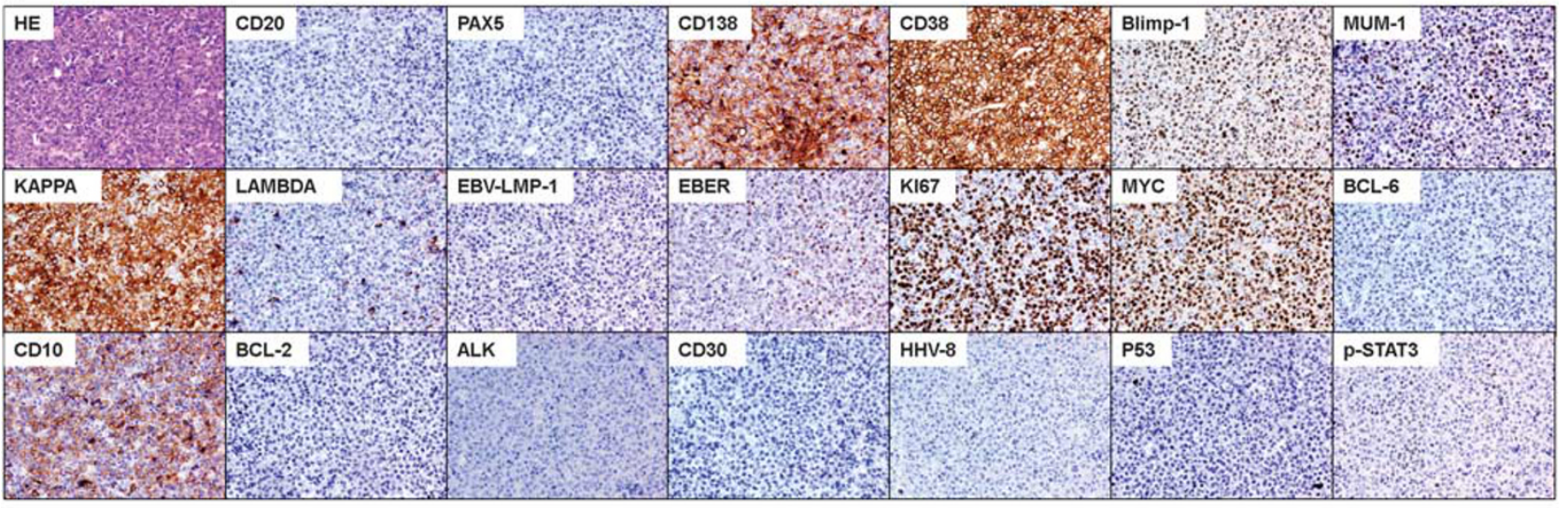

c

d

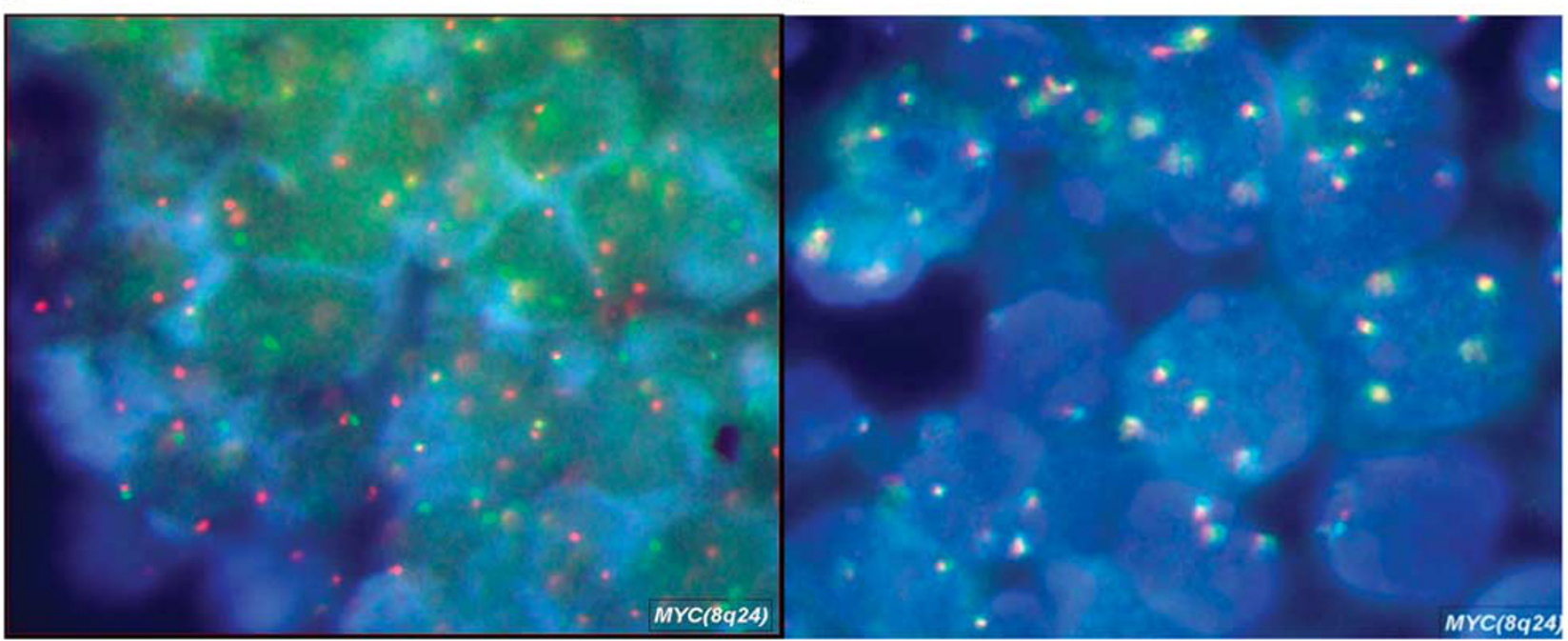

Figure 1 (a and b) Characteristic phenotypic profile of plasmablastic lymphoma cases with absence of expression of B-cell markers (CD20, PAX5) and overexpression of plasma cell-related antigens (Blimp1, CD38, and CD138). Note the homogeneous expression of proliferation markers (Ki67) and MYC protein. Absence of expression of HHV-8 and ALK are required for the exclusion of other lymphoma types. Note that some cases may express CD10 (Table 1). (c) Fluorescent in situ hybridization for MYC rearrangements using a break apart probe. A split signal is clearly identified in a significant fraction of the nuclei. This pattern was found in 12 cases (46\%). (d) Multiple copies of the MYC gene are found in a significant fraction of the nuclei, consistent with gains of MYC locus. This pattern was found in 3 cases (11\%). 


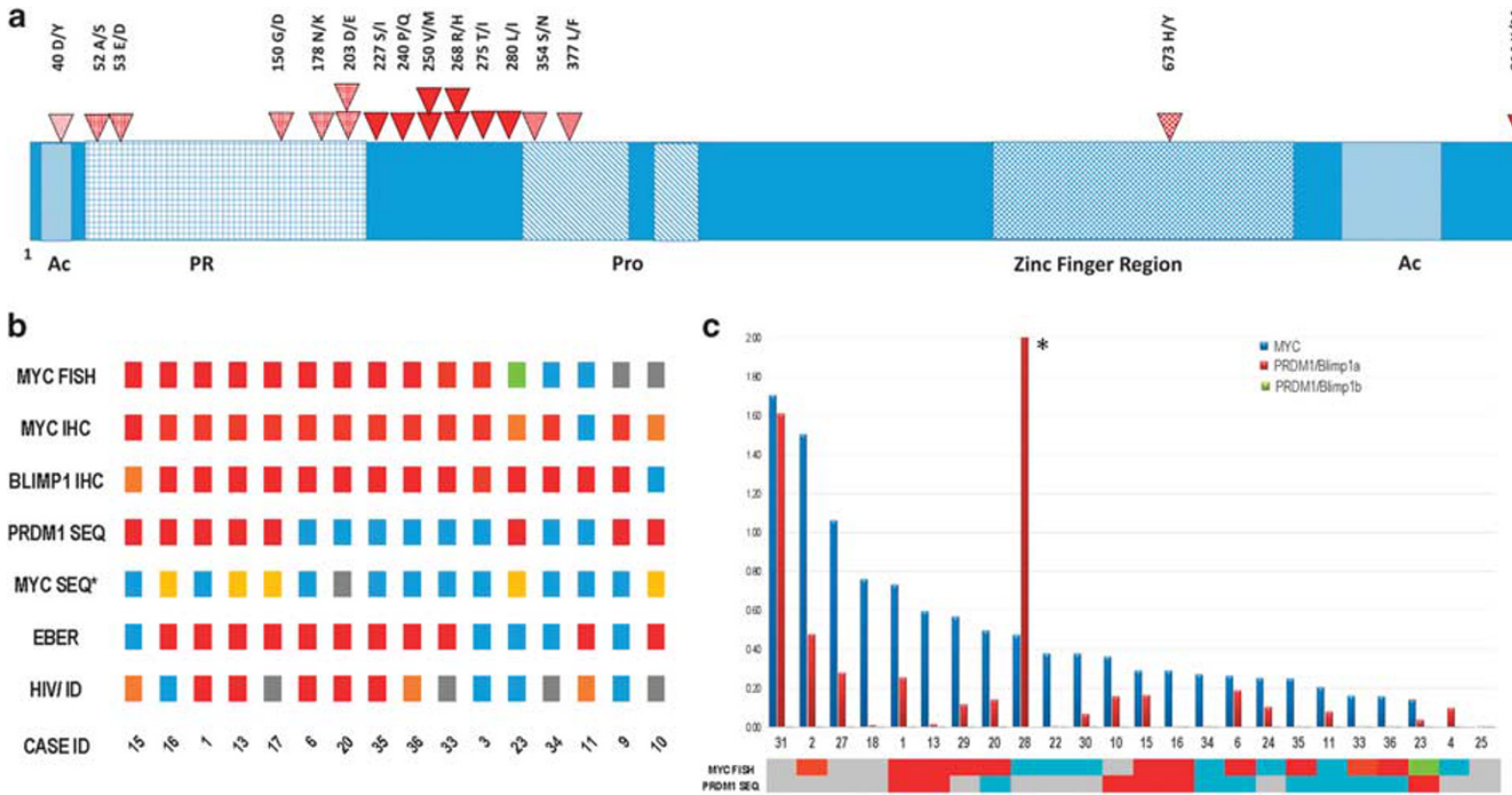

Figure 2 (a) Schematic diagram of PRDM1 gene illustrating the different functional domains and the location of the variants here identified. Eight out of $16(50 \%)$ cases analyzed had at least one missense variation in PRDM1-exonic regions (range 1-6 variations). Three of these variations were recurrent, one of these located in the PR region of the gene (c. 843, $\mathrm{C}>\mathrm{G}$, D203E) in exon 4, and two involving exon 5 (c982 G>A, V250M and c1037G > A, R268H). Other variations concentrate in PR (c388 G> T, A52S; c393 G> T, E53D; c683 G>A, G150D; c768 C>A, N178K), Pro (c1295 G>A, S354N, c1365G>T, L377F), Zn-finger (c2251 C> T, H673Y), and acidic regions in the N-terminal (c352 G> T, D40Y). (b) Figure showing the coincidence of PRDM1 mutations with other relevant variables such as fluorescent in situ hybridization for MYC rearrangements, and MYC- and Blimp1-protein expression, EBV positivity by EBER, and immunodeficiency status. Mutations in PRDM1 were found in $56 \%$ of the plasmablastic lymphoma cases with MYC translocations by fluorescent in situ hybridization, but was not restricted to those cases. MYC and Blimp1 proteins are coexpressed in the majority of the cases (80\%), irrespective of $M Y C$ translocation status. (c) Histogram showing the RT-PCR $\left(2^{-\delta C t}\right)$ values for MYC, PRDM1 $\alpha$, and PRDM1 $\beta$ in a subset of 24 cases. MYC transcripts are consistently expressed in the majority of the cases, concomitant to PRDM1 $\alpha$ mRNA. PRDM1 $\beta$ mRNA expression is not detected. Except for one case $\left({ }^{*}\right)$, MYC transcripts have a higher level of expression than PRDM1 $\alpha$ transcripts. The presence of PRDM1 mutations do not abolish the expression of PRDM1 $\alpha$ transcripts and low-level expression leads to detectable protein expression by immunohistochemistry in all but one mutated case (case no. 10, mutated in exon 2, see 2C and Table 2). MYC fluorescent in situ hybridization: red, translocated; orange, gained; green, deleted; blue, normal; gray, not analyzed. MYC immunohistochemistry, Blimp1 immunohistochemistry: color scale representation of levels of protein expression by immunohistochemistry (red: $100 \%$ positive, blue: $100 \%$ negative). PRDM1 seq: red identifies cases with missense variants in PRDM1 gene. MYC seq*: yellow identifies cases with silent mutations in the promoter region of $M Y C$ gene. EBER: red identifies cases positive for EBV-EBERs chromogenic in situ hybridization. HIV/ID identifies different immunodeficiency states: red denotes HIV, light orange identifies cases with other sources of immunodeficiency (post-transplant setting or therapy for previous lymphoproliferative disorder), and blue identifies cases without identifiable cause for immunodeficiency. Ac, acidic domain; PR, PR domain; Pro, proline rich domain.

Blimp1 protein binds to repress $M Y C$ expression ${ }^{30}$ ), 7 silent variants were found in 5 out of 15 cases (33\%; Supplementary Figure 1).

\section{Plasmablastic Lymphoma Cases Express High Levels of MYC mRNA Together with Blimp1 $\alpha$ mRNA Isoform}

Quantitative RT-PCR analysis showed that plasmablastic lymphoma cases overexpress homogeneously MYC mRNA, consistently with protein overexpression observed with immunohistochemistry. As Blimp1 clone here used identifies both Blimp1 isoforms (clone ROS195 recognizes a site between amino acids 176 and 307, unpublished data from G Roncador, CNIO monoclonal antibody Unit), we performed RT-PCR to evaluate whether a differential expression between isoforms occurs in plasmablastic lymphoma cases. Here, we have found that plasmablastic cases express high levels of PRDM1 $\alpha$ isoform transcripts and almost negligible amounts of PRDM1 $\beta$. In conclusion, Blimp1-protein expression detected in plasmablastic lymphoma cases is related to PRDM1 $\alpha$ transcripts. Interestingly, enough both PRDM1 $\alpha$ isoform and MYC mRNA are coexpressed in the majority of cases, consistent with the pattern found by immunohistochemistry (Figure 2).

PRDM1 Gene is Recurrently Mutated in Plasmablastic Lymphoma and These Mutations Cooperate with MYC Dysregulation

Twenty gene variants were identified in 8 cases after targeted amplicon-based next-generation sequencing of PRDM1 gene. Thus, 8 out of $16(50 \%)$ cases analyzed had at least 1 missense variation in PRDM1-exonic regions (range 1-6 variations). Three 
Table 2 Summary of the PRDM1 missense variants and mutations found in 8 out of 16 cases analyzed by targeted exonic next-generation sequencing

\begin{tabular}{|c|c|c|c|c|c|c|c|c|c|}
\hline Case ID & Location & Domain & Allele & cDNA position & Codons & $A A$ & Change & Consequence $^{*}$ & Existing variation \\
\hline 1 & Exon 5 & & $\mathrm{~T}$ & 1058 & aCa/aTa & 275 & $\mathrm{~T} / \mathrm{I}$ & Missense/neutral & dbSNP rs777939985 \\
\hline 1 & Exon 5 & Pro & $\mathrm{T}$ & 1365 & $\mathrm{ttG} / \mathrm{ttT}$ & 377 & $\mathrm{~L} / \mathrm{F}$ & Missense/neutral & - \\
\hline 1 & Exon 5 & & A & 1037 & cGt/cAt & 268 & $\mathrm{R} / \mathrm{H}$ & Missense/neutral & COSM3154292 somatic \\
\hline 9 & Exon 4 & PR & G & 843 & $\mathrm{gaC} / \mathrm{gaG}$ & 203 & $\mathrm{D} / \mathrm{E}$ & Missense/neutral & dbSNP rs811925* \\
\hline 10 & Exon 2 & Ac & $\mathrm{T}$ & 352 & $\mathrm{Gac} / \mathrm{Tac}$ & 40 & $\mathrm{D} / \mathrm{Y}$ & Missense/deleterious & - \\
\hline 13 & Exon 4 & $\mathrm{PR}$ & A & 683 & gGc/gAc & 150 & G/D & Missense/deleterious & - \\
\hline 15 & Exon 5 & & A & 1037 & cGt/cAt & 268 & $\mathrm{R} / \mathrm{H}$ & Missense/neutral & COSM3154292 somatic \\
\hline 16 & Exon 4 & $\mathrm{PR}$ & G & 843 & gaC/gaG & 203 & $\mathrm{D} / \mathrm{E}$ & Missense/neutral & dbSNP rs811925* \\
\hline 16 & Exon 5 & Pro & A & 1295 & $\mathrm{aGc} / \mathrm{aAc}$ & 354 & $\mathrm{~S} / \mathrm{N}$ & Missense/neutral & dbSNP rs143040512* \\
\hline 16 & Exon 7 & Zn finger & $\mathrm{T}$ & 2251 & $\mathrm{Cac} / \mathrm{Tac}$ & 673 & $\mathrm{H} / \mathrm{Y}$ & Missense/deleterious & - \\
\hline 16 & Exon 5 & & A & 982 & Gtg/Atg & 250 & $\mathrm{~V} / \mathrm{M}$ & Missense/deleterious & COSM3748876 somatic \\
\hline 17 & Exon 5 & & $\mathrm{~T}$ & 914 & $\mathrm{aGt} / \mathrm{aTt}$ & 227 & S/I & Missense/neutral & - \\
\hline 17 & Exon 5 & & A & 982 & Gtg/Atg & 250 & $\mathrm{~V} / \mathrm{M}$ & Missense/deleterious & COSM3748876 somatic \\
\hline 17 & Exon 5 & & A & 1072 & $\mathrm{Ctc} / \mathrm{Atc}$ & 280 & $\mathrm{~L} / \mathrm{I}$ & Missense/neutral & - \\
\hline 17 & Exon 7 & & $\mathrm{~T}$ & 2675 & aAg/aTg & 814 & $\mathrm{~K} / \mathrm{M}$ & Missense/deleterious & - \\
\hline 17 & Exon 7 & & A & 2708 & $\mathrm{cCt} / \mathrm{cAt}$ & 825 & $\mathrm{P} / \mathrm{H}$ & Missense/deleterious & - \\
\hline 17 & Exon 4 & PR & A & 768 & aaC/aaA & 178 & N/K & Missense/deleterious & - \\
\hline 23 & Exon 2 & $\mathrm{PR}$ & $\mathrm{T}$ & 388 & Gct/Tct & 52 & $\mathrm{~A} / \mathrm{S}$ & Missense/neutral & - \\
\hline 23 & Exon 2 & $\mathrm{PR}$ & $\mathrm{T}$ & 393 & gaG/gaT & 53 & $\mathrm{E} / \mathrm{D}$ & Missense/neutral & - \\
\hline 23 & Exon 5 & & A & 953 & $\mathrm{cCa} / \mathrm{cAa}$ & 240 & $\mathrm{P} / \mathrm{Q}$ & Missense/neutral & - \\
\hline
\end{tabular}

Exonic location, cDNA position, single-nucleotide change observed, and amino acid change predicted, together with predicted consequence using three different algorithms is shown. In addition, dbSNP and COSMIC id is provided when available. Changes identified with * have been previously reported in DLBCL samples according to the literature.

of these variations were recurrent, one of these located in the PR region of the gene (c. $843, \mathrm{C}>\mathrm{G}$, D203E) in exon 4 and two involving exon 5 (c982 G > A, V250M and c1037G > A, R268H). Other variations concentrate in PR (c388 G>T, A52S; c393 G> T, E53D; c683 G>A, G150D; c768 C>A, N178K), Pro (c1295 G>A, S354N, c1365G>T, L377F), Znfinger (c2251 $\mathrm{C}>\mathrm{T}, \mathrm{H} 673 \mathrm{Y}$ ), and acidic regions in the N-terminal (c352 G>T, D40Y). Of all variants found, eight mutations were considered deleterious and damaging by at least three different algorithms used (Polyphen, SIFT, and Condel). See a summary of the mutations found in Table 2 and Figure 2. No frameshift in/del or splice-site mutations were found, probably due, at least partially to the exontargeted approach to next-generation sequecing. Two mutations affecting exon 5 (c982 G>A, V250M and c1037G > A, R268H) have been confirmed somatic in cases of urothelial carcinoma (COSM3748876 and COSM3154292, respectively). Two of these variants have been previously considered SNPs in the literature (c1295 G>A, S354N and c. 843, C>G, $\mathrm{D} 203 \mathrm{E})^{22}$ and were considered neutral by prediction algorithms. Another variant (c1058 C> T, 275T/I) is considered a SNP by dbSNP (rs777939985) and is considered neutral by prediction algorithms.

Correlation of data from mutational analysis with fluorescent in situ hybridization for $M Y C$ rearrangements, MYC and Blimp1 proteins, and transcript expression was performed. As shown in Figure 2, all nine cases with MYC translocation had MYC overexpression by RT-PCR and immunohistochemistry. A total of 5 out of these 9 cases had a second-genetic hit with PRDM1 mutations. All nine cases had Blimp1-protein overexpression and all except three had significant expression by RT-PCR of PRDM1 $\alpha$ isoform. Of the five cases with PRDM1 mutations, two cases had reduced levels of PRDM1 $\alpha$ transcript, suggesting an impaired transcription but detectable protein levels.

In two cases, $M Y C$ gains were detected by fluorescent in situ hybridization, and correlated with MYC-protein and transcript overexpression, and Blimp1-protein expression, in the absence of genetic mutations in PRDM1. One of these cases had reduced levels of PRDM1 $\alpha$ transcripts. Three out of 5 additional cases had PRDM1 mutations in the absence of $M Y C$ rearrangements (in two cases, $M Y C$ rearrangements were not evaluable and could not be excluded). Interestingly enough, 1 out of these 5 cases had MYC and Blimp1 proteins, and mRNA expression associated with $M Y C$ deletion by fluorescent in situ hybridization and PRDM1 mutations in PR region in exon 2 (c388 G>T, A52S; c393 G>T, E53D). c388 G>T, A52S mutation is considered deleterious by Condel algorithm. Of note, the levels of transcript expression for both MYC and PRDM1 $\alpha$ were reduced in comparison with the other cases (Figure 2).

Interestingly, PRDM1 missense mutations are found as second-genetic hit in a significant fraction (5 cases, 56\%) of plasmablastic lymphoma cases with $M Y C$ translocation, but importantly, are not restricted to $M Y C$-translocated cases. In three of our PRDM1-mutated cases, MYC was found to be deleted (one case) or not analyzed (two cases). 


\section{Discussion}

Here, we describe a new series of 36 plasmablastic lymphoma cases. In our series, plasmablastic lymphoma cases are grouped in three well-defined clinical categories: cases associated with HIV infection, cases associated with other sources of immunosuppression such as post-transplant setting or immunodeficiency related to treatment for previous lymphoid malignancy, and cases without a demonstrable cause of immunodeficiency. Cases with HIV infection have a significant younger age than patients in the category without demonstrable immunodeficiency. The median age of patients in the latter category is 66 years of age. Thus, a significant proportion of plasmablastic lymphoma cases (20 out of 36 in our series) are age-related and may be associated with age-related immunodeficiency. These findings are similar to those reported in a recently published large series of 61 plasmablastic lymphoma $\operatorname{cases}^{13}$ and in a large meta-analysis on the topic. ${ }^{5}$ Thus, age-related plasmablastic lymphoma cases are increasingly recognized in the literature and in opposition to other lymphoma types thought to be associated with immunosenescence (ie, EBV-positive DLBCL NOS, formerly known as EBVpositive DLBCL of the elderly); plasmablastic lymphoma cases are not always associated with EBV positivity. Limited data in the previously published series suggest that EBV-negative cases in older patients have the worse outcome ${ }^{5,13,31,32}$ encouraging further biological insights on that subgroup of patients.

Plasmablastic lymphoma cases disclose a phenotypical profile consistent with a terminal B-cell differentiation program, with downregulation of B-cell markers and overexpression of plasma cellassociated markers such as CD138, CD38, and Blimp1. This protein expression profile has been described extensively in previous papers ${ }^{4}$ and confirms recently published data on the gene expression profile of plasmablastic lymphoma cases. ${ }^{9}$ Furthermore, this protein expression profile is common to other B-cell lymphomas with immunoblastic/plasmablastic differentiation such as ALK+ large B-cell lymphoma. ${ }^{12}$ However, other proteins found to be overexpressed in ALK+ large B-cell lymphoma such as STAT3-P ${ }^{12}$ have only a limited expression in our plasmablastic lymphoma cases.

Interestingly, EBV by EBER was found positive in $58 \%$ of our cases, similar to other case series previously published. It is becoming widely accepted that EBV positivity is not a requirement for the diagnosis of plasmablastic lymphoma. Furthermore, recently published gene expression profiling data suggest that the transcriptomic profile of plasmablastic lymphoma cases is not affected by either EBV presence or HIV infection by the patient, ${ }^{9}$ consistent with the notion that both HIV-associated, immunodeficiency-associated, and age-related plasmablastic lymphoma cases are defined by the same phenotype in the neoplastic cells.

Regarding Blimp1 expression, we have further clarified that in plasmablastic lymphoma cases, Blimp1-protein expression is related to PRDM1 $\alpha$ full-length transcripts. In our subset of 24 samples studied by RT-PCR, we found virtually undetectable levels of PRDM1 $\beta$ transcripts. These data suggest that plasmablastic lymphoma cases usually express the full-length protein, unlike myeloma cell lines (U266 and NCI-H929) ${ }^{33}$ and OCI-Ly3 DLBCL cell line that aberrantly express the defective PRDM1 $\beta$ isoform. ${ }^{34}$

$M Y C$ translocation is the main cytogenetic abnormality that we found in our cases, together with $M Y C$ gains. These data are consistent with previous results in the literature showing the presence of $M Y C$ translocations and gains in plasmablastic lymphoma cases. ${ }^{10-12}$ However, to the best of our knowledge, this is the first time that $M Y C$ deletion is reported in a case of plasmablastic lymphoma. Interestingly enough, plasmablastic lymphoma cases usually lack concomitant translocations in BCL2 and BCL6, not qualifying for double-hit B-cell lymphoma as conventionally recognized. ${ }^{10,35,36}$ A single case report has demonstrated concomitant presence of BCL2 and $M Y C$ translocation in a case of plasmablastic-like transformation from low-grade follicular lymphoma. ${ }^{37}$

We have found in all our 36 cases overexpression of MYC protein. This overexpression is homogeneous and involves the majority of the neoplastic cells in the majority of the cases $(91 \%$ of the cases had $>40 \%$ of positive cells) in concordance with the $M Y C$ transcript levels found in the subset of 24 cases studied by RT-PCR. The overexpression of MYC protein is found in both $M Y C$-translocated and -amplified cases (12 and 3 cases, respectively), as well as cases with deletion of $M Y C$ (1 case) or absence of $M Y C$ gene abnormalities by fluorescent in situ hybridization (10 cases). These data are in agreement with recently published data that suggest that MYC-protein overexpression in plasmablastic lymphoma is the rule, irrespective of the presence of translocations or other genetic alterations in $M Y C$ gene. ${ }^{9,12,13}$ In a proportion of cases, MYC dysregulation might be related to $M Y C$ translocation or gain (46\% in our series), but this leaves around $50 \%$ of the cases without a clear biological basis for $M Y C$ overexpression.

Interestingly, we have found coexpression of MYC and Blimp1 proteins in $80 \%$ of our cases. This pattern of coexpression is also found at the mRNA level regarding MYC and PRDM1 $\alpha$ transcripts (Figure 2). This pattern of coexpression of MYC and Blimp1 is highly unexpected based on previously published knowledge on the regulation of MYC expression by Blimp1 in normal plasma cells. ${ }^{15,17,30}$ This led us to investigate whether mutations in $M Y C$ promoter and/or PRDM1 gene may explain the coexpression of MYC and Blimp1. 
Our data show that recurrent missense mutations in PRDM1 gene are common in plasmablastic lymphoma cases $(50 \%$ of cases in our series). PRDM1-mutation pattern is consistent with the observed in other tumor suppressor genes, with multiple mutations along the gene, clustered in the half N-terminal portion of the protein in this case. Furthermore, PRDM1 mutations target critical functional domains (PR motif, proline rich domain, acidic region, and DNA-binding Zn-finger domain) involved in the regulation of targets such as $M Y C,{ }^{30}$ CIITA, ${ }^{38}$ and PAX5. ${ }^{39}$ Interestingly enough, in plasmablastic lymphoma cases, unlike DLBCL lymphoma cases, ${ }^{21}$ these mutations do not abolish PRDM1 $\alpha$ transcript and protein expression, and this may explain why the cases show the characteristic plasma cell phenotype.

Thus, PRDM1 mutations might impair the regulatory functions of PRDM1 gene without affecting its terminal B-cell differentiation-related functions. This differential effect might be related to the characteristic pattern of mutations along PRDM1 gene, affecting regions involved in target-gene repression such as PR domain. These effects recapitulate the phenotype observed by the expression of the PRDM1 $\beta$ isoform, with limited capacity to repress target genes but conserved DNA-binding activity, nuclear localization, and association with histone deacetylases and deacetylase activity. ${ }^{33}$ However, our RT-PCR data clearly demonstrate that PRDM $1 \beta$ is not expressed in our cases and only full-length PRDM1 $\alpha$ transcripts are found. These data collectively imply that PRDM $1 \alpha$ transcripts might be functionally impaired by the missense mutations here found, affecting exclusively the regulatory functions of PRDM1 gene.

To the best of our knowledge, this is the first time that somatic mutations in PRDM1 gene have been described in plasmablastic lymphoma cases. Furthermore, this is the first gene known to be affected by somatic mutations in these uncommon B-cell lymphoma entity.

Previous reports have described a variety of PRDM1 mutations in a significant fraction of conventional DLBCL and primary central nervous system lymphoma cases. ${ }^{14,20-22,28}$ These mutations are common in ABC-type DLBCL, and together with PRDM1 deletion lead to inactivation of the gene and downregulation of the protein expression. ${ }^{21,22}$ These studies suggest that the genetic inactivation of PRDM1 may lead to a block in terminal B-cell differentiation, essential to the pathogenesis of ABC-type DLBCL. ${ }^{21}$

In plasmablastic lymphoma cases, however, our data suggest that PRDM1 mutations do not impair terminal B-cell differentiation, but may contribute to the oncogenicity of $M Y C$, usually dysregulated by $M Y C$ translocation or MYC amplification. PRDM1 mutation may serve as a second-genetic hit, in a tumor suppressor gene that synergizes with $M Y C$ owing to the impaired $M Y C$-regulatory capacity of
PRDM1 mutants. ${ }^{30}$ In conclusion, aberrant coexpression of MYC and PRDM1 $\alpha$ owing to the genetic changes is responsible for the phenotype of plasmablastic lymphoma cases.

\section{Acknowledgments}

This study was supported by grants from the Ministerio de Sanidad y Consumo (PI051623, PI052800, CP06/00002, and RTICC) Asociación Española contra el Cancer (AECC) and the Ministerio de Ciencia e Innovación (SAF 2008-03871), Spain. Santiago Montes-Moreno has received a grant from Ministerio de Economía y competitividad, MBAE BA15/00053.

\section{Disclosure/conflict of interest}

The authors declare no conflict of interest.

\section{References}

1 Stein H, Harris NL, Campo E. Plasmablastic lymphoma. In: Swerdlow SH, Campo E, Harris NL et al (eds). WHO Classification of Tumours of Haematopoietic and Lymphoid Tissues. IARC Press: Lyon, France, 2008, pp 256-257.

2 Campo E, Swerdlow SH, Harris NL, et al. The 2008 WHO classification of lymphoid neoplasms and beyond: evolving concepts and practical applications. Blood 2011;117:5019-5032.

3 Delecluse HJ, Anagnostopoulos I, Dallenbach F, et al. Plasmablastic lymphomas of the oral cavity: a new entity associated with the human immunodeficiency virus infection. Blood 1997;89:1413-1420.

4 Montes-Moreno S, Gonzalez-Medina AR, RodriguezPinilla SM, et al. Aggressive large B-cell lymphoma with plasma cell differentiation: immunohistochemical characterization of plasmablastic lymphoma and diffuse large B-cell lymphoma with partial plasmablastic phenotype. Haematologica 2010;95:1342-1349.

5 Morscio J, Dierickx D, Nijs J, et al. Clinicopathologic comparison of plasmablastic lymphoma in HIV-positive, immunocompetent, and posttransplant patients: single-center series of 25 cases and meta-analysis of 277 reported cases. Am J Surg Pathol 2014;38:875-886.

6 Castillo JJ, Bibas M, Miranda RN. The biology and treatment of plasmablastic lymphoma. Blood 2015;125: 2323-2330.

7 Teruya-Feldstein J, Chiao E, Filippa DA, et al. CD20negative large-cell lymphoma with plasmablastic features: a clinically heterogenous spectrum in both HIVpositive and -negative patients. Ann Oncol 2004;15: 1673-1679.

8 Colomo L, Loong F, Rives S, et al. Diffuse large B-cell lymphomas with plasmablastic differentiation represent a heterogeneous group of disease entities. Am J Surg Pathol 2004;28:736-747.

9 Chapman J, Gentles AJ, Sujoy V, et al. Gene expression analysis of plasmablastic lymphoma identifies downregulation of B-cell receptor signaling and additional unique transcriptional programs. Leukemia 2015;29: 2270-2273. 
10 Valera A, Balagué O, Colomo L, et al. IG/MYC rearrangements are the main cytogenetic alteration in plasmablastic lymphomas. Am J Surg Pathol 2010;34: 1686-1694.

11 Taddesse-Heath L, Meloni-Ehrig A, Scheerle J, et al. Plasmablastic lymphoma with MYC translocation: evidence for a common pathway in the generation of plasmablastic features. Mod Pathol 2010;23:991-999.

12 Valera A, Colomo L, Martinez A, et al. ALK-positive large B-cell lymphomas express a terminal B-cell differentiation program and activated STAT3 but lack MYC rearrangements. Mod Pathol 2013;26:1329-1337.

13 Loghavi S, Alayed K, Aladily TN, et al. Stage, age, and EBV status impact outcomes of plasmablastic lymphoma patients: a clinicopathologic analysis of 61 patients. J Hematol Oncol 2015;8:65.

14 Pasqualucci L, Compagno M, Houldsworth J, et al. Inactivation of the PRDM1/BLIMP1 gene in diffuse large B cell lymphoma. J Exp Med 2006;203:311-317.

15 Shaffer AL, Lin KI, Kuo TC, et al. Blimp-1 orchestrates plasma cell differentiation by extinguishing the mature B cell gene expression program. Immunity 2002;17: 51-62.

16 Maestre L, Tooze R, Canamero M, et al. Expression pattern of XBP1(S) in human B-cell lymphomas. Haematologica 2009;94:419-422.

17 Lin Y, Wong K, Calame K. Repression of c-myc transcription by Blimp-1, an inducer of terminal B cell differentiation. Science 1997;276:596-599.

18 Pasqualucci L, Trifonov V, Fabbri G, et al. Analysis of the coding genome of diffuse large B-cell lymphoma. Nat Genet 2011;43:830-837.

19 Zhang J, Grubor V, Love CL, et al. Genetic heterogeneity of diffuse large B-cell lymphoma. Proc Natl Acad Sci USA 2013;110:1398-1403.

20 Tam W, Gomez M, Chadburn A, et al. Mutational analysis of PRDM1 indicates a tumor-suppressor role in diffuse large B-cell lymphomas. Blood 2006;107: 4090-4100.

21 Mandelbaum J, Bhagat G, Tang H, et al. BLIMP1 is a tumor suppressor gene frequently disrupted in activated B cell-like diffuse large B cell lymphoma. Cancer Cell 2010;18:568-579.

22 Courts C, Montesinos-Rongen M, Brunn A, et al. Recurrent inactivation of the PRDM1 gene in primary central nervous system lymphoma. J Neuropathol Exp Neurol 2008;67:720-727.

23 Schwindt H, Vater I, Kreuz M, et al. Chromosomal imbalances and partial uniparental disomies in primary central nervous system lymphoma. Leukemia 2009;23:1875-1884.

24 Pasqualucci L, Dalla-Favera R. SnapShot: diffuse large B cell lymphoma. Cancer Cell 2014;25:132-e1.

25 Chapuy B, Cheng H, Watahiki A, et al. Diffuse large B-cell lymphoma patient-derived xenograft models capture the molecular and biologic heterogeneity of the disease. Blood 2016;127:2203-2213.

26 Kallioniemi OP, Wagner U, Kononen J, et al. Tissue microarray technology for high-throughput molecular profiling of cancer. Hum Mol Genet 2001;10:657-662.

27 Robinson JT, Thorvaldsdottir H, Winckler W, et al. Integrative genomics viewer. Nat Biotechnol 2011;29: 24-26.

28 Tate G, Hirayama-Ohashi Y, Kishimoto K, et al. Novel BLIMP1/PRDM1 gene mutations in B-cell lymphoma. Cancer Genet Cytogenet 2007;172:151-153.

29 Horn H, Staiger AM, Vohringer M, et al. Diffuse large B-cell lymphomas of immunoblastic type are a major reservoir for MYC-IGH translocations. Am J Surg Pathol 2015;39:61-66.

$30 \mathrm{Yu}$ J, Angelin-Duclos C, Greenwood J, et al. Transcriptional repression by blimp-1 (PRDI-BF1) involves recruitment of histone deacetylase. Mol Cell Biol 2000;20:2592-2603.

31 Liu F, Asano N, Tatematsu A, et al. Plasmablastic lymphoma of the elderly: a clinicopathological comparison with age-related Epstein-Barr virus-associated B cell lymphoproliferative disorder. Histopathology 2012;61:1183-1197.

32 Castillo JJ, Winer ES, Stachurski D, et al. Clinical and pathological differences between human immunodeficiency virus-positive and human immunodeficiency virus-negative patients with plasmablastic lymphoma. Leuk Lymphoma 2010;51:2047-2053.

33 Gyory I, Fejer G, Ghosh N, et al. Identification of a functionally impaired positive regulatory domain I binding factor 1 transcription repressor in myeloma cell lines. J Immunol 2003;170:3125-3133.

34 Tam W, Gomez M, Nie K. Significance of PRDM1beta expression as a prognostic marker in diffuse large B-cell lymphomas. Blood 2008;111:2488-2489.

35 Aukema SM, Siebert R, Schuuring E, et al. Double-hit B-cell lymphomas. Blood 2011;117:2319-2331.

36 Swerdlow SH. Diagnosis of 'double hit' diffuse large B-cell lymphoma and B-cell lymphoma, unclassifiable, with features intermediate between DLBCL and Burkitt lymphoma: when and how, FISH versus IHC. Hematology Am Soc Hematol Educ Program 2014;2014:90-99.

37 Ouansafi I, He B, Fraser C, et al. Transformation of follicular lymphoma to plasmablastic lymphoma with c-myc gene rearrangement. Am J Clin Pathol 2010;134: 972-981.

38 Ghosh N, Gyory I, Wright G, et al. Positive regulatory domain I binding factor 1 silences class II transactivator expression in multiple myeloma cells. J Biol Chem 2001;276:15264-15268.

39 Lin KI, Angelin-Duclos C, Kuo TC, et al. Blimp-1dependent repression of Pax-5 is required for differentiation of $\mathrm{B}$ cells to immunoglobulin M-secreting plasma cells. Mol Cell Biol 2002;22:4771-4780.

Supplementary Information accompanies the paper on Modern Pathology website (http://www.nature.com/ modpathol) 